\title{
THAT QUASINILPOTENT OPERATORS ARE NORM-LIMITS OF NILPOTENT OPERATORS REVISITED
}

\author{
C. APOSTOL, C. FOIAŞ AND C. PEARCY
}

\begin{abstract}
A new short proof is given that every quasinilpotent operator on a separable, infinite dimensional, complex Hilbert space is a norm-limit of nilpotent operators.
\end{abstract}

Let $\mathcal{H}$ be a separable, infinite dimensional, complex Hilbert space, and let $\mathcal{E}(\mathcal{H})$ denote the algebra of all bounded linear operators on $\mathcal{H}$.

In [2] it was shown that every quasinilpotent operator $T$ in $\mathcal{L}(\mathcal{H})$ (i.e., every $T$ whose spectrum is the singleton $\{0\}$ ) is a norm-limit of nilpotent operators. The purpose of this note is to give a very short and easily remembered proof of this theorem. Our proof has two basic ingredients: the use of a model for quasinilpotent operators constructed in [4], and the use of a deep theorem of Voiculescu [7]. For completeness we review the relevant facts from [4] and [7] that we shall need.

Let $\mathcal{H}_{\infty}=\mathcal{H} \oplus \mathcal{H} \oplus \ldots$ be the direct sum of $S_{0}$ copies of $\mathcal{H}$ indexed by the positive integers, let $\kappa=\left\{\kappa_{n}\right\}_{n=1}^{\infty}$ be a monotone decreasing sequence of nonnegative numbers converging to zero, and let $U_{\kappa}$ denote the quasinilpotent backward weighted shift operator in $\mathcal{L}\left(\mathcal{H}_{\infty}\right)$ defined by the equation

$$
U_{\kappa}\left(x_{1}, x_{2}, \ldots, x_{n}, \ldots\right)=\left(\kappa_{1} x_{2}, \kappa_{2} x_{3}, \ldots, \kappa_{n} x_{n+1}, \ldots\right) \text {. }
$$

THEOREM A [4]. If $T$ is any quasinilpotent operator in $\mathcal{L}(\mathcal{H})$, then there is a sequence $\kappa=\kappa(T)=\left\{\kappa_{n}\right\}_{n=1}^{\infty}$ with the above described properties and a subspace $\mathfrak{N}$ of $\mathcal{H}_{\infty}$ that is invariant under the quasinilpotent operator $U_{\kappa}$ such that $T$ is similar to $U_{\kappa} \mid \mathfrak{R}$ and such that the subspace $\mathcal{H}_{\infty} \ominus \mathfrak{R}$ is infinite dimensional.

We shall need some lemmas, the first two of which are completely elementary.

LEMMA 1. Let $T_{1}$ and $T_{2}$ belong to $\mathcal{L}\left(\mathcal{H}_{1}\right)$ and $\mathcal{L}\left(\mathcal{H}_{2}\right)$, respectively, and suppose that there exists a sequence $\left\{S_{n}\right\}_{n=1}^{\infty}$ of invertible operators $S_{n}: \mathcal{H}_{1} \rightarrow$ $\mathcal{H}_{2}$ such that $\left\|S_{n}^{-1} T_{1} S_{n}-T_{2}\right\| \rightarrow 0$. If $T_{1}$ is a norm-limit of nilpotent operators, then so is $T_{2}$.

LEMMA 2. The operator $U_{\kappa}$ in (1) is a norm-limit of nilpotent operators in $\mathcal{L}\left(\mathcal{H}_{\infty}\right)$ (since the sequence $\kappa$ converges to zero).

Received by the editors February 21, 1978 and, in revised form, April 11, 1978.

AMS (MOS) subject classifications (1970). Primary 47A65.

Key words and phrases. Quasinilpotent operator, norm-limit. 
The following lemma is only slightly less elementary and is a special case of [6, Proposition 6.18].

LEMma 3. Let $S=\left(S_{i j}\right)_{i, j=1}^{n}$ be an $n \times n$ matrix with operator entries acting on the direct sum $\mathcal{H} \oplus \cdots \oplus \mathcal{H}$ of $n$ copies of $\mathcal{H}$ and having the property that $S_{i j}=0$ if $i>j$. If each $S_{i i}, 1 \leqslant i \leqslant n$, is a norm-limit of nilpotent operators, then so is $S$. On the other hand, if $S$ is a norm-limit of nilpotent operators, then so is $S_{11} \oplus \cdots \oplus S_{n n}$.

Proof. For $1 \leqslant i \leqslant n$, let $N_{i i}$ be a nilpotent operator such that $\left\|N_{i i}-S_{i i}\right\|$ $<\varepsilon$. Then the matrix $\tilde{S}$ obtained by replacing each diagonal entry $S_{i i}$ by the corresponding operator $N_{i i}$ satisfies $\|\tilde{S}-S\|<\varepsilon$, and it is easy to see that $\tilde{S}$ is nilpotent. To go the other way, observe that by choosing $n$ sufficiently large, the matrix

$$
\operatorname{Diag}\left(1, n, \ldots, n^{n-1}\right)\left(S_{i j}\right) \operatorname{Diag}\left(1, \frac{1}{n}, \ldots, \frac{1}{n^{n-1}}\right)
$$

can be made as close as desired to $S_{11} \oplus \cdots \oplus S_{n n}$, and use Lemma 1 .

LEMMA 4. If $T$ belongs to $\mathcal{L}(\mathcal{H})$ and is quasinilpotent, and Theorem $\mathrm{A}$ is used to write the space $\mathcal{H}_{\infty}$ as $\mathcal{H}_{\infty}=\mathfrak{N} \oplus \mathfrak{K}^{\perp}$ where $U_{\kappa} \mathfrak{N} \subset \mathfrak{K}$ and $T$ is similar to $T^{\prime}=U_{\kappa} \mid \Upsilon_{R}$, then in the corresponding matrix decomposition

$$
U_{\kappa}=\left(\begin{array}{ll}
T^{\prime} & A \\
0 & S
\end{array}\right),
$$

the operator $S$ is quasinilpotent and $T^{\prime} \oplus S$ is a norm-limit of nilpotent operators.

Proof. That $S$ is quasinilpotent follows from the fact that $U_{\kappa}$ is quasinilpotent (Theorem A) and the form of the matrix (2). That $T^{\prime} \oplus S$ is a norm-limit of nilpotent operators is an immediate consequence of Lemmas 2 and 3.

THEOREM B [1]. If $T$ is any quasinilpotent operator in $\mathcal{L}(\mathcal{H})$, then $T \oplus 0$ is a norm-limit of nilpotent operators in $\mathcal{L}(\mathcal{H} \oplus \mathcal{H})$.

Proof. According to Lemmas 4 and 1, $T \oplus S$ is a norm-limit of nilpotent operators on $\mathcal{H} \oplus \mathcal{H}$ and $S$ is quasinilpotent. Thus, by a theorem of Rota (cf. [5, p. 77]), $S$ is similar to operators of arbitrarily small norm, and the result follows by another application of Lemma 1.

COROLlary 1. Suppose $T_{1}$ and $T_{2}$ are quasinilpotent operators in $\mathcal{L}(\mathcal{H})$ and both $T_{1}$ and $T_{2}^{*}$ have infinite dimensional kernels. Then $T_{1} \oplus T_{2}$ is the normlimit of nilpotent operators in $\mathcal{L}(\mathcal{H} \oplus \mathcal{H})$.

Proof. Up to unitary equivalence, $T_{1} \oplus T_{2}$ may be written as a $4 \times 4$ matrix 


$$
\left(\begin{array}{ll|ll}
0 & B_{1} & 0 & 0 \\
0 & C_{1} & 0 & 0 \\
\hline 0 & 0 & 0 & 0 \\
0 & 0 & B_{2} & C_{2}
\end{array}\right) .
$$

acting on the Hilbert space $\mathcal{H} \oplus \mathscr{H} \oplus \mathscr{H} \oplus \mathscr{H}$, and this matrix, in turn, is unitarily equivalent to

$$
\left(\begin{array}{ll|ll}
C_{2} & 0 & 0 & B_{2} \\
0 & 0 & B_{1} & 0 \\
\hline 0 & 0 & C_{1} & 0 \\
0 & 0 & 0 & 0
\end{array}\right) .
$$

Since $C_{1}$ and $C_{2}$ are quasinilpotent, the corollary now follows from Theorem $B$ and Lemma 3.

We turn now to some preparatory notation that we shall need to review the above-mentioned theorem of Voiculescu. If $T_{1}$ and $T_{2}$ are operators in $\mathcal{L}\left(\mathcal{F G}_{1}\right)$ and $\mathcal{L}\left(\mathcal{H}_{2}\right)$, respectively, and if there exist a unitary operator $U: \mathcal{H}_{1} \rightarrow \mathscr{K}_{2}$ and a compact operator $K$ in $\mathcal{L}\left(\mathcal{H}_{2}\right)$ such that $U T_{1} U^{*}=T_{2}+K$ and such that $\|K\|<\varepsilon$, then we say that $T_{1}$ and $T_{2}$ are $\varepsilon$-compalent and we write $T_{1} \sim T_{2}(\varepsilon)$. Observe that the relation of $\varepsilon$ - compalence is symmetric, that is, $T_{1} \sim T_{2}(\varepsilon)$ if and only if $T_{2} \sim T_{1}(\varepsilon)$. We denote the ideal of compact operators in $\mathcal{E}(\mathcal{H})$ by $\mathbf{K}(\mathcal{H})$, and the quotient map of $\mathcal{L}(\mathcal{H})$ onto the Calkin algebra $\mathcal{E}(\mathcal{H}) / \mathbf{K}(\mathcal{H})$ by $\pi$. If $T \in \mathcal{L}(\mathcal{H})$, we denote by $C_{e}^{*}(T)$ the separable $C^{*}$-subalgebra of $\mathcal{L}(\mathcal{H}) / \mathbf{K}(\mathcal{H})$ generated by $\pi(T)$ and $\pi\left(1_{\mathcal{H}}\right)$.

TheORem C [7]. Let $T \in \mathcal{L}(\mathcal{H})$, and let $\rho$ be a faithful representation of $C_{e}^{*}(T)$ on a separable Hilbert space $\mathscr{K}$ such that $\rho\left(\pi\left(1_{\mathscr{C}}\right)\right)=1_{\mathscr{K}}$. Then for every positive number $\varepsilon, T \oplus \rho(\pi(T)) \sim T(\varepsilon)$.

Based on the above considerations we are now prepared to give a transparent proof of the following theorem.

THEOREM D [2]. Every quasinilpotent operator $T$ in $\mathcal{L}(\mathcal{H})$ is a norm-limit of nilpotent operators.

Proof. Using Theorem $C$ and Lemma 1, we observe that it suffices to construct some faithful representation $\rho$ of $C_{e}^{*}(T)$ on a separable Hilbert space $\mathscr{K}$ such that $\rho\left(\pi\left(1_{\mathcal{C}}\right)\right)=1_{\mathcal{K}}$ and such that $T \oplus \rho(\pi(T))$ is a norm-limit of nilpotent operators in $\mathcal{E}(\mathcal{H} \oplus \mathscr{K})$. Furthermore, if $\tau$ is a faithful representation of $C_{e}^{*}(T)$ on a separable Hilbert space $\Re$ such that $\tau\left(\pi\left(1_{\Im}\right)\right)=1_{\mathscr{V}}$ then $\mathcal{K}$ may be taken to be $\Re \oplus \Re$ and $\rho$ to be $\tau \oplus \tau$. Thus it suffices to find a representation $\tau$ with the above-mentioned properties such that $[T \oplus$ $\tau(\pi(T))] \oplus \tau(\pi(T))$ is a norm-limit of nilpotent operators. On the other hand, by virtue of Corollary 1 , it suffices to construct such a representation $\tau$ with the property that both $\tau(\pi(T))$ and $\tau\left(\pi\left(T^{*}\right)\right)$ have infinite dimensional kernels, and this goes as follows. Since $T$ and $T^{*}$ are quasinilpotent, one 
knows from [8] (cf. also [3, Theorem 3.1]) that there exist projections $E$ and $F$ in $\mathcal{L}(\mathcal{H})$ of infinite rank such that $\pi(T) \pi(E)=0$ and $\pi\left(T^{*}\right) \pi(F)=0$. Furthermore, $E$ may be written as $E=\sum_{n=1}^{\infty} E_{n}$, where $\left\{E_{n}\right\}$ is an orthogonal sequence of projections each of which has infinite rank, and similarly $F$ may be written as $F=\sum_{n=1}^{\infty} F_{n}$. Let $Q$ be the (separable) $C^{*}$-subalgebra of $\mathcal{E}(\mathcal{H}) / \mathbf{K}(\mathcal{H})$ generated by $\pi(T), \pi\left(1_{\mathcal{H}}\right)$, and the countable families $\left\{\pi\left(E_{n}\right)\right\}_{n=1}^{\infty}$ and $\left\{\pi\left(F_{n}\right)\right\}_{n=1}^{\infty}$, and let $\nu$ be a faithful representation of $\mathcal{Q}$ on a separable Hilbert space $\Re$ so chosen that $\nu\left(\pi\left(1_{\mathscr{C}}\right)\right)=1_{\mathscr{C}}$. Then $\nu(\pi(T)) \nu\left(\pi\left(E_{n}\right)\right)=0$ and $\nu\left(\pi\left(T^{*}\right)\right) \nu\left(\pi\left(F_{n}\right)\right)=0$ for all positive integers $n$, so clearly the operators $\nu(\pi(T))$ and $\nu\left(\pi\left(T^{*}\right)\right)=(\nu(\pi(T)))^{*}$ have infinite dimensional kernels, and the result follows by setting $\tau=\nu \mid C_{e}^{*}(T)$.

\section{REFERENCES}

1. C. Apostol, On the norm-closure of nilpotents, Rev. Roumaine Math. Pures Appl. 19 (1974), 277-282.

2. C. Apostol and D. Voiculescu, On a problem of Halmos, Rev. Roumaine Math. Pures Appl. 19 (1974), 283-284.

3. A. Brown and C. Pearcy, Compact restrictions of operators, Acta Sci. Math. (Szeged) 32 (1971), 271-282.

4. C. Foias and C. Pearcy, A model for quasinilpotent operators, Michigan Math. J. 21 (1974), 399-404.

5. P. R. Halmos, A Hilbert space problem book, Van Nostrand, Princeton, N. J., 1967.

6. C. Pearcy, Some recent developments in operator theory, CBMS Regional Conf. Ser. in Math., no. 36, Amer. Math. Soc., Providence, R. I., 1978.

7. D. Voiculescu, A noncommutative Weyl-von Neumann theorem, Rev. Roumaine Math. Pures Appl. 21 (1976), 97-113.

8. F. Wolf, On the essential spectrum of partial differential boundary problems, Comm. Pure Appl. Math. 12 (1959), 211-228.

DePARTMENT OF MATHEMATICS, INCREST, Bucharest, RumaNiA

Department of Mathematics, University of Bucharest, Bucharest, Rumanta

Departmeint of Mathematics, University of Michigan, Ann Arbor, Michigan 48109 